\title{
Features of the adaptation of the indigenous people of the Arctic
}

\author{
Evgeniya. Nikolaeva ${ }^{1, *}$, Olga Kolosova ${ }^{2}$, and Elena Belchusova ${ }^{2}$ \\ ${ }^{1}$ M.K. Ammosov North-Eastern Federal University, 27, Oyunsky street, Yakutsk, 677013 Russia. \\ ${ }^{2}$ The Yakut Scientific Centre of the SBRAS, Institution of the Russian Academy of Sciences, 41, Lenin avenue, Yakutsk, \\ 677007, Russia
}

\begin{abstract}
To determine age-specific differences in adaptation of indigenous residents, nonspecific adaptive body reactions (NARs) have been studied among the Evenki men aged 13 to 86, permanently living in the Arctic. The results indicated the presence of age-specific differences in NARs. The decline of nonspecific body resistance in every fourth teenager is correlated with adaptive reactions developing in parallel with underlying low-level body reactivity. Adaptive reactions in men aged 75-90 were characterized by a medium to a high reactivity level, which enabled preservation of body reserves even under extreme climate conditions. Two age groups (2235 and 61-74) included the highest percentages of people with adverse NARs. Therefore, men in these age groups should be considered as a major risk group for developing both psychosomatic and somatic diseases.
\end{abstract}

\section{Introduction}

Development of some specific patterns of the physiological function in individuals arriving to live in the Arctic is defined by the region's extreme climate, geography, and heliogeophysical conditions. At the bottom-line of an adaptive response to changing environment conditions (including extreme climate factors), there are nonspecific adaptive body reactions (NARs). The study of strategies for adapting to the specific ecological and climatic factors of the body of the indigenous people of the Arctic, who in the process of evolution developed specific mechanisms for biochemical and physiological adaptation, is extremely important and necessary. Previous studies have led to the conclusion that there are adaptive types in the population of people that are adapted to well-defined climatic and geographical conditions of existence. Adaptive types of human organisms determine survival and can also be constraints in possible adaptive processes in new environmental conditions [1]. The effectiveness of adaptation to extreme conditions depends not only on genetic factors, but also on the morpho-physiological parameters of a person, which are formed in ontogenesis under certain climatic and geographical conditions [2-7].

In the literature, views on NARs are closely related to the study of adaption mechanisms. According to the theory of Hans Selye about a 'general adaptation syndrome', a state of stress (strain) can be induced by any strong stimulus (stressor). Because stimuli affecting the body may be of various intensity, theory of NARs was further developed by L.KH. Garkavi and colleagues
(1968). The body, reacting to stimuli of varying strength and quality, causing fluctuations in the parameters of homeostasis, tries to keep them within optimal boundaries, using a common periodic system of nonspecific, anti-stressor reactions of the body. With long-term exposure to a complex of extreme factors in the body, in the process of evolution, the most optimal adaptive reactions are fixed, which allow a person not only to survive in these conditions, but to fully live, work, develop, etc. Under the influence of factors of varying strength and intensity, regardless of their nature, physiological and biochemical changes take place in the body, leading to nonspecific adaptive reactions of the organism. Following this recent theory, the system of human adaptive reactions includes, alongside with the stress reaction (SR) and over-activation (OA) reaction, the following types of reaction: training reaction (TR) developing under weak stimuli; activation reaction (AR) subdivided into quiet activation reaction (QAR) and advanced activation reaction (AAR) developing under moderate stimuli. Each type of adaptive reaction corresponds to a specific set of indicators describing neuro-endocrine and immune body subsystems, processes of metabolism and energy metabolism, and mental status. Good adaptation to changing environmental conditions can be achieved if there is a sufficient adaptation potential of a body. This capability is conditioned not just by the presence of a functional body reserve, but also by body reaction adequacy and economy, as well as efficiency of control over functional reserve expenditure and recovery [7-10]. In addition, adaptive reactions can be correlated with different body

* Corresponding author: en.nikolaeva@mail.ru 
reactivity levels (RL), depending on the magnitude of the stimulus. The hallmark of the high reactivity level is low sensitivity threshold in response to factors of minor magnitude, while a lower reactivity level is associated with growing sensitivity threshold developing under factors of large magnitude. Apparently, the tendency is such that a higher body reactivity level correlates with more effective synchronization of body's protective systems due to multiplicity of minor interactions. A decline in the reactivity level is associated with the onset and escalation of the signs of tension, which signalize desynchronized functioning of body systems. Thus, reactions of training, quiet activation, and advanced activation alongside with underlying a high reactivity level are considered beneficial body reactions. Unfavourable NARs, which pave the way for predisease, include an over-activation (OA) reaction, stress reaction, and reactions of training, quiet, and advanced activation developing in parallel with the low body reactivity level.

In the course of evolution, under certain climatic and geographic factors, native northern minorities, living in the harsh Arctic environment from generation to generation, phenotypically developed specific adaptation mechanisms, which formed the basis for optimal functioning and regulation of body systems [2,6,8]. Since genetically fixed adaptive mechanisms in the process of ontogenesis are manifested depending on the living conditions, it is very important to understand their phenotypic manifestation in different age groups of the same ethnic group living for a long time in the Arctic region.

In light of the above said, it was deemed relevant to study the age-related features of the ecological and physiological adaptation mechanisms developing in residents of the Arctic in response to extreme environmental factors.

The aim of this study was to detect patterns in the development of nonspecific adaptive reactions in indigenous males (Evenki) residing permanently in the Arctic.

\section{Material and methods}

259 men, permanently residing in Olenek Evenki National Region, were observed. They volunteered to be included to the study. All patients were Evenki by ethnicity, aged 13 to 86 . All were divided to 6 age groups: 13-16 (A1); 17-20 (A2); 21-34 (A3); 36-60 (A4); 61-74 (A5); 75 or more (A6).

Types of NARs and reactivity levels (RL) were determined based on L.KH. Garkavi et al. Fasting peripheral blood samples were obtained in the morning; blood smears were prepared according to the standard technique.

To assess the vegetative regulation, the method of heart rate variability analysis (HRV) according to R.M. Baevsky was used. The study was conducted on the hardware-software complex "VNS-Micro" company Neurosoft (Ivanovo) for 5 minutes in the prone position in the morning. Using a heart rate variability analysis based on processing data on the distribution of cardio intervals, a stress index of regulatory systems (Stress Index - SI) was determined, which reflects the degree of centralization of heart rhythm control. The method of identifying the hidden periodicity of the dynamic range of cardiointervals determined the power spectrum in the ranges:

1. High frequencies (High Frequency - HF) - 0.15$0.40 \mathrm{~Hz}$.

2. Low frequencies (Low Frequency - LF) - 0.04$0.15 \mathrm{~Hz}$.

3. Very low frequencies (Very Low Frequency VLF) - 0.003-0.04 Hz.

The study is based on a simple random sample. Storage of research results and primary processing of the material was carried out in the original database Microsoft Excel 2007. Statistical processing was made using conventional methods with the package "SPSS 10". Mean (M), mean square deviation (SD) and mean (m) error were determined. The nature of the distribution was assessed using the Kolmogorov-Smirnov criterion. In the normal distribution of variables, the student's unpaired t-test was used to determine the differences between the two independent groups, and in the nonparametric one, the Wilcoxon - Mann-Whitney test was used. Correlation analysis methods for parametric and nonparametric types of distribution - Pearson and Spearman criteria, respectively - were used to identify the relationship between the studied parameters. Differences and correlations were considered significant at $p<0.05$. The study is complied with ethical guidelines of World Medical Association Declaration of Helsinki and 'The fundamentals of the legislation of the Russian Federation on the protection of citizens' health' (issued in 1993).

\section{Results and discussion}

The results of studying the state of adaptation processes of the organism of indigenous small ethnic groups of the Arctic region, regardless of age, indicate that more than half of men $(54 \%)$ show a high level of reactivity in the body, ensuring the possibility of rapid mobilization of functions and an adequate response of the body to external influences (Fig. 1).

Here is the structure of the types of nonspecific adaptive reactions (A) and (B) reactivity level (UR) of the body of Evenk men $(\mathrm{p}=259 ; \%)$ :

Against this background, a rather high percentage of people $(30 \%)$ is found in a state of low reactivity characterized by a predominance of inhibitory processes in the body, both at the level of the central nervous system and at the periphery, whose body is unable to develop an adequate, rapid response of the adaptive reaction. The adverse types of NARs (RS and OA) of the body of Evenki men are 14\%. Despite the high percentage of men with favourable NARs (TR, QAP and AAR) $(86 \%)$, taking into account the fact that $30 \%$ of them occur at low RL, it can be said that $35 \%$ of other people in the body have a mismatch functioning of the 
subsystems of the body, which is the cause of the intensity of adaptive systems

A

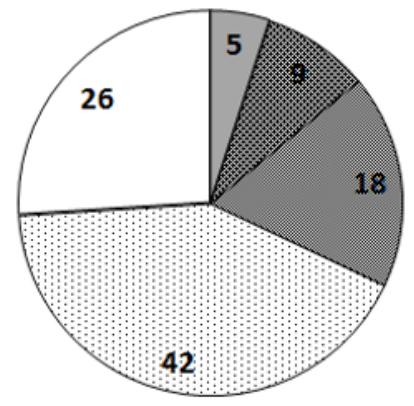

口RS :OA IAAR DQAR DTR

B

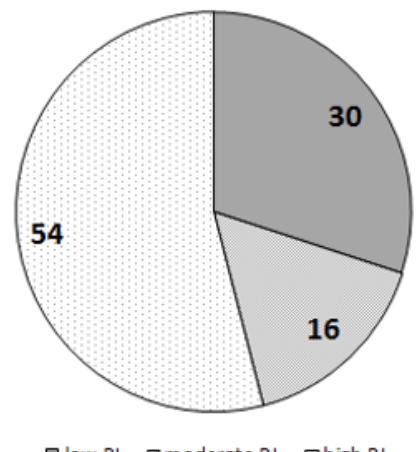

Fig. 1. Structure of types of non-specific adaptive reactions (A) and the level of reactivity (B) of the body of Evenki men $(\mathrm{n}=159, \%)$ : RS - stress reaction, RT-training reaction, RSAquiet activation reaction, RPA-increased activation reaction, $\mathrm{OA}$ - over-activation reaction.

A more detailed study of the structure of adaptive reactions of the organism was carried out in 6 age groups (Fig. 2). Statistically meaningful differences $(p<0.05)$ were demonstrated in the adaptive response patterns between study groups.

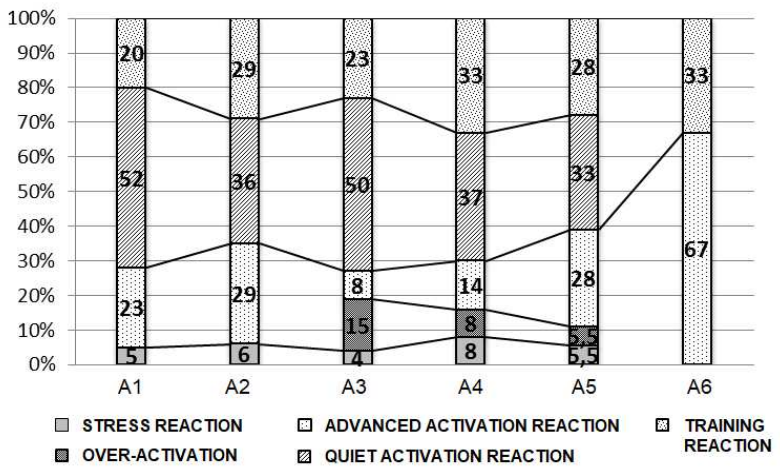

Fig. 2. Prevalence of nonspecific adaptive body reaction indicators in males in relation to age $(\%)(\mathrm{A} 1$ - 13-16 years, A2 - 17-20 years, A3 - 21-34 years, A4 - 35-60 years, A5 61-74 years, A6 - $75-90$ years)

In age group A1, activation reactions prevailed in the majority: QAR and AAR (52\% and $23 \%$, respectfully), suggesting high level of health, increased activity of regulatory and protective body subsystems, predomination of anabolism with moderate energy expenditure, indicating that adaptive responses are developing in a favourable way. TR was observed in $20 \%$ of adolescents, associated with the presence of protective inhibition in central nervous system (CNS), decreased catabolism, accumulation and preservation of plasticity and energy reserves of the body. TR in adult age would be labelled as an anti-stressor recovery reaction, but in adolescent age, with its intensive physical growth and mental development, extensive changes affecting hormonal and nervous control processes, this signals a worsened health level. Moreover, 5\% of adolescents were assessed to be in a stressed state.

The study of body reactivity levels showed that in $29 \%$ of Evenki adolescents, adaptive reactions were associated with the low or moderate reactivity level, suggesting a decline in sensitivity of CNS, desyncronization in regulatory systems, and consequently, tensed adaptive reactions (Fig. 3).

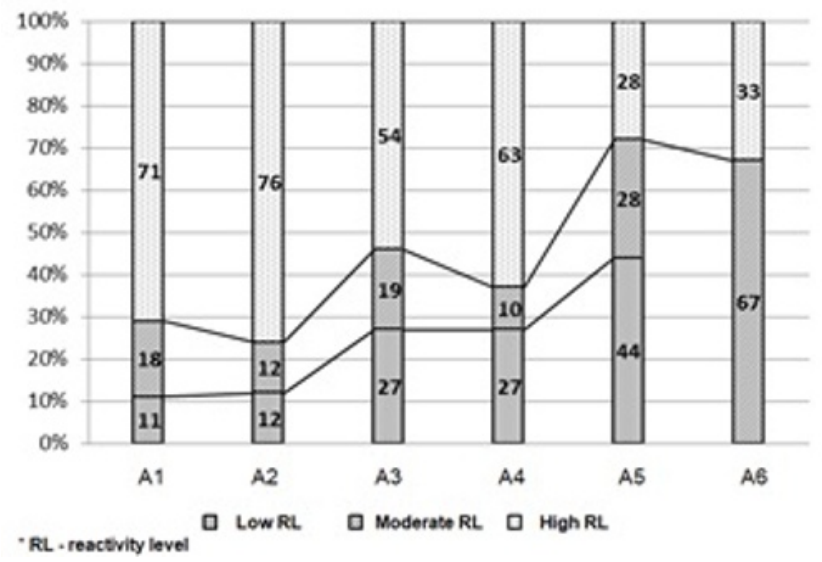

Fig. 3. Prevalence of reactivity levels of nonspecific adaptive body reaction indicators in males in relation to age (\%) (A1 13-16 years, A2 - 17-20 years, A3 - 21-34 years, A4 - 35-60 years, A5 - 61-74 years, A6 - 75 - 90 years).

In adolescence (A2), the proportion of individuals with QAR was significantly (by $16 \%$ ) smaller $(\mathrm{p}<0.05)$ and proportion of those with TR was 9\% larger compared to A1 group. Aside from experiencing physiological changes, life of adolescents is filled with social events of utmost importance such as graduating from school, entering university, starting an independent life, etc. A compound effect of climate, social, psychological factors may, probably, be overwhelming and excessive for Evenki teenagers, leading to development of protective inhibition in CNS and training reaction.

Among men in the early middle age (A3), AAR was observed in $21 \%$ less than it was in A2 group. Overactivation reaction was demonstrated in $15 \%$, which may indicate that adaptation mechanisms were tensed, and AAR was transitioning to OA reaction. Compared to A2 group, there were twice more $(\mathrm{p}<0.01)$ men with a low body reactivity level. At the low reactivity level, adaptive reactions develop following stimuli of strong magnitude, which is a sign of diminished CNS sensitivity, leading eventually to desynchronization of humoral and neural control mechanisms, increased 
energy expenditures, and reduced functional body reserve.

In groups $\mathrm{A} 4, \mathrm{~A} 5$, and $\mathrm{A} 6$, the number of men demonstrating QAR gradually decreased with aging, while people in this group increasingly show AAR, which speaks for favourable development of adaptation mechanisms. The maximum number of persons with unfavourable NAR is observed in age group A3. In subsequent groups, the number of people with unfavourable NAR is gradually decreasing (Fig. 4).

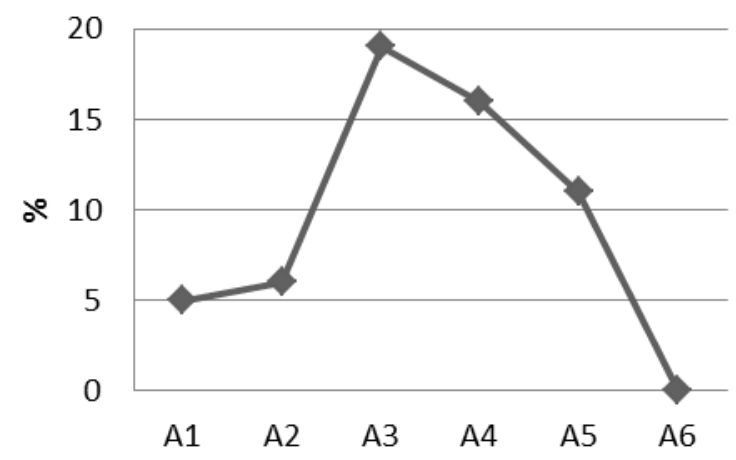

Fig. 4. Dynamics of unfavourable nonspecific adaptive body reaction indicators in males in relation to age (\%) (A1 - 13-16 years, A2 - 17-20 years, A3 - 21-34 years, A4 - 35-60 years, A5 - 61-74 years, A6 - $75-90$ years).

But at the elderly age (A5), the proportion of individuals with high reactivity levels declined dramatically $(\mathrm{p}<0.05)$, alongside with the increase (more than $50 \%$ higher) in the number of men with low level reactivity. As far as an average life span in male population of northern regions is less than 64 years, it could be deduced that Evenki men making it to this age would have exhausted functional body reserve, energy and protective body capacities, so that the body would fail to adequately respond to any stimuli. And this finally could be leading to irreversible desynchronizing deleterious processes.

In senile men, AAR and TR develop in association with moderate or high reactivity levels, which is why individuals with high adaptive body reserve are more likely to reach this age.

Thus, the largest percentage of people with unfavorable NARs are men aged (22-35) years and (6174) years.

Since, as it is supposed, mobilization of energy reserves and development of the corresponding NARs in the body occurs as a result of a change in the level of activity of regulatory systems, a study was conducted concerning the stress index (SI) of the regulatory systems of men in adult age groups (Fig. 5). In a healthy person in central Russia in a calm state, the magnitude of the SI is $60-120 \mathrm{cu}$. In our study, in three age groups (A3, A4, and A5), the SI value was significantly (p $<0.05)$ higher than the standard indicators. The high voltage of the state of regulatory systems in the men of the Evenks forms unfavorable NARs.

The acrophase SI falls on the age group A3, and the level of tension of the regulatory systems in comparison with the A2 group increases significantly $(\mathrm{p}<0.01)$ by more than 7.7 times.

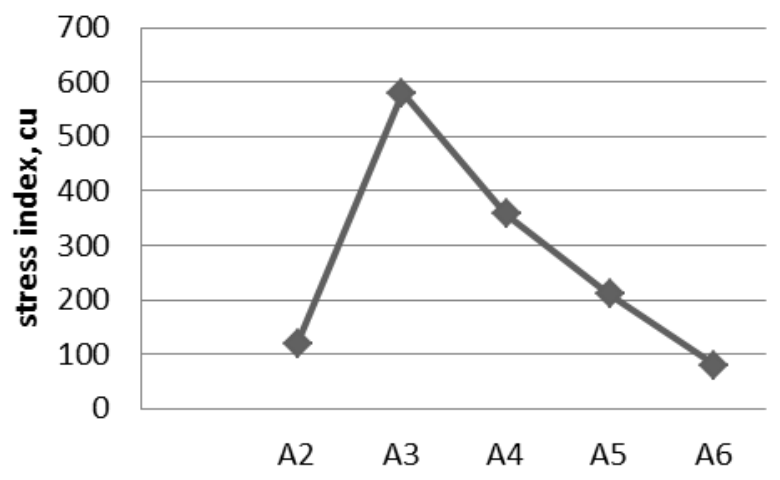

Fig. 5. The magnitude of the stress index of the body's regulatory systems in males in relation to age (cu), (A2 - 17-20 years, A3 - 21-34 years, A4 - 35-60 years, A5 - 61-74 years, A6 - $75-90$ years).

The physiological mechanisms of the formation of unfavorable NAR in the age category from 21 to 34 years (A3) are associated with increased duplication of adaptation processes by the central regulation. This is noted in cases where the body, when a voltage condition of regulatory systems occurs, constantly suffers a shortage of functional reserves. The results obtained to identify the spectrum of regulation of functions in men indicate that in the age group A3 almost $47 \%$ occupy a very low frequency range (VLF\%). The power of this range is associated with the central neuro-humoral regulation, which corresponds to the third, highest, uneconomic level of activity - the level of central, intersystem control, which causes the highest level of SI.

The formation of unfavorable NAR in the A5 age group is based on somewhat different mechanisms; in particular, this is associated with an increase in the tone of the sympathetic division of the autonomic nervous system. The power of the low frequency range (LF\%) rises, which is a marker of the sympathetic nervous system and corresponds to the second level of activity the level of intrasystem regulation.

\section{Conclusion}

Study results indicate the presence of age-specific differences in NARs observed among Evenki men permanently residing in the Arctic. Decline of nonspecific body resistance in every fourth teenager correlated with adaptive reactions developing in parallel with underlying low-level body reactivity. Adaptive reactions in men of the senile age group were characterized by medium to the high reactivity level, which enabled preservation of body reserves, even under extreme climate conditions. It was established that in two age groups - early adulthood ((21-35) years) and old age ((61-74) years), the highest level of tension of adaptive reactions occurs due to low recovery and mobilizing potential, immunodepression, desynchronization of functional and stress-realizing 
systems of the organism, the predominance of catabolism processes on the background of transcendental arousal (leading to transcendent inhibition) of the central nervous system. The physiological mechanisms of NAR formation in these groups differ in different levels of regulatory systems and, therefore, in the magnitude of the body's functioning tension. In group A5, the formation of NAR is carried out by intrasystemic rearrangements, by increasing the tone of the sympathetic division of the autonomic nervous system. In the age group $\mathrm{A} 3$, the mechanism of the formation of adaptation reactions is based on energetically extremely costly processes of intersystem rearrangements, due to the increased duplication of adaptation processes by the central regulation.

\section{Acknowledgement}

The study was carried out within the framework of the projects: "Study of chronophysiological, molecular mechanisms of adaptation and psychoemotional stress of a person under the conditions of the combined action of extreme climatic, technogenic factors in the Arctic and Subarctic and a pandemic, post-pandemic, development of ways to increase the adaptive potential" 121021600269-0), Federal Research Center YSC SB RAS and "Physiological and biochemical mechanisms of adaptation of plants, animals, humans to the conditions of the Arctic / Subarctic and the development of biological products based on natural northern raw materials that increase the efficiency of the adaptation process and the level of human health in extreme environmental conditions" (No. 0297 - 2021-0025 registration number AAAA-A21-121012190035-9) of the Institute for Biological Problems of Permafrost, Federal Research Center of YSC SB RAS.

\section{References}

1. J. Launay, G. Savourey, Ind Health 47 (2009)

2. T.M. Mäkinen, Am J Hum Biol 19 (2007)

3. T.M. Mäkinen, Front Biosci - Scholar 2 (2010)

4. J. Bittel, Int J Sports Med 13 (1992)

5. A.T. Steegmann, Jr. Human, Am J Hum Biol 19 (2007)

6. A.J. Young, M.J, Fregly, C.M. Blatteis. Handbook of physiology. Section 4: Environmental physiology. Oxford University Press (Oxford, UK, 1996)

7. N.I. Douglas, E.N. Nikolaeva, N.S. Baisheva, O.N. Kolosova, E.A. Belchusova, L.E. Vasilieva, Int J of Biomed 9 (2019)

8. T. Adams, B.G. Covino. J Appl Physiol 12 (1958)

9. W.D. van Marken Lichtenbelt, H.A. Daanen, Curr Opin Clin Nutr Metab Care. 6 (2003)

10. J.W. Castellani, A.J. Young, Auton Neurosci. 196 (2016) 